\title{
Écarts régionaux dans le fardeau économique attribuable au surplus de poids, à la sédentarité et à l'usage du tabac en Colombie-Britannique
}

\author{
H. Krueger, Ph. D. (1, 2); J. M. Koot, B. Sc. (2); D. P. Rasali, Ph. D. (3, 4); S. E. Gustin, M.P.H. (3); M. Pennock, M. Sc. (5)
}

Cet article a fait l'objet d'une évaluation par les pairs.

Diffuser cet article sur Twitter

\section{Résumé}

Introduction : Les taux de prévalence du surplus de poids, de l'usage du tabac et de la sédentarité varient sensiblement d'une région à l'autre en Colombie-Britannique (C.-B.). La présente étude vise à déterminer la portée d'une éventuelle réduction du fardeau économique en C.-B. si toutes les régions de la province atteignaient des taux de prévalence équivalents à ceux de la région dont les taux sont les plus bas pour ces trois facteurs de risque.

Méthodes : Nous avons utilisé une approche élaborée précédemment fondée sur la fraction étiologique du risque pour estimer le fardeau économique associé aux divers facteurs de risque. Le risque relatif selon le sexe et les données de prévalence selon l'âge et le sexe ont été utilisés dans la modélisation.

Résultats : Le fardeau économique annuel attribuable à ces trois facteurs de risque en C.-B. s'élevait à environ 5,6 milliards de dollars en 2013, la proportion la plus élevée de ce total étant attribuable au surplus de poids (2,6 milliards), suivie de celle de l'usage du tabac ( 2 milliards). Même si la C.-B. possède des taux de prévalence de ces facteurs de risque plus bas que toute autre province canadienne, il existe d'importants écarts en son sein. Si chaque région de la province devait atteindre les taux de prévalence les plus bas pour les trois facteurs de risque, un fardeau économique de 1,4 milliard (24\% du total de 5,6 milliards) pourrait être supprimé annuellement.

Conclusion : Il existe des disparités notables dans la prévalence de chacun des facteurs de risque au sein des régions sanitaires de la C.-B., qui se reflètent dans le fardeau économique attribuable à chaque région. Un éventail de facteurs sociaux, environnementaux et économiques expliquent probablement une partie de ces écarts géographiques, et ces facteurs sous-jacents devraient être pris en compte lors de la mise en place de programmes de prévention.

Mots-clés : fardeau économique de la maladie, populations à risque, facteurs de risque, usage du tabac, activité physique, poids

\section{Introduction}

Le fardeau économique annuel du surplus de poids, de la sédentarité et de l'usage du tabac s'élevait à environ 52,8 milliards en
2013 au Canada ${ }^{1}$. Une réduction relative annuelle modeste de $1 \%$ de la prévalence de ces trois facteurs de risque peut avoir une incidence importante sur la santé et sur l'économie au fil du temps au niveau

\section{Points saillants}

- En Colombie-Britannique en 2013, le fardeau économique attribuable au surplus de poids (2,6 milliards) était plus élevé que celui attribuable à l'usage du tabac (2 milliards) ou à la sédentarité (1 milliard).

- Le fardeau économique du surplus de poids, de la sédentarité et de l'usage du tabac varie entre les 16 régions sanitaires de la ColombieBritannique.

- La réduction de la prévalence du surplus de poids, de la sédentarité et de l'usage du tabac dans l'ensemble des régions sanitaires au niveau de celle de la région possédant les taux les plus bas permettrait de réduire le fardeau économique annuel total d'un quart, en le faisant passer de 5,6 milliards à 4,2 milliards.

- L'écart dans la prévalence du surplus de poids, de la sédentarité et de l'usage du tabac est plus élevé au sein de la C.-B. (24\%) qu'entre les provinces $(10 \%)$.

- La reonnaissance de ces variations spatiales entre les régions sanitaires pourrait contribuer à décider quels efforts de prévention devraient être orientés vers quelles régions.

de la population, entraînant une réduction annuelle d'environ 8,5 milliards du fardeau économique du Canada d'ici $2031^{2}$.

Rappelons qu'avec son territoire de près de 10 millions de kilomètres carrés, le

Rattachement des auteurs :

1. School of Population and Public Health, University of British Columbia, Vancouver (Colombie-Britannique), Canada

2. H. Krueger and Associates Inc., Delta (Colombie-Britannique), Canada

3. Population and Public Health, British Columbia Provincial Health Services Authority (PHSA), Vancouver (Colombie-Britannique), Canada

4. Faculty of Kinesiology and Health Studies, University of Regina, Regina (Saskatchewan), Canada

5. Population Health Surveillance and Public Health Planning, British Columbia Ministry of Health, Vancouver (Colombie-Britannique), Canada

Correspondance: Hans Krueger, H. Krueger and Associates Inc., 4554 48B Street, Delta, (C.-B.) V4K 2R8; tél. : 604-946-5464; courriel : hans@krueger.ca 
Canada est le deuxième pays au monde en termes de superficie. Le pays est divisé en 10 provinces et 3 territoires. La population totale s'élevait à environ 35,2 millions en 2013, celle des provinces variant de 146000 à l'île-du-Prince-Édouard à 13,6 millions en Ontario ${ }^{3}$. La Colombie-Britannique (C.-B.), la province la plus à l'ouest, a une population de 4,7 millions.

Parmi toutes les provinces, la prévalence de l'usage du tabac, du surplus de poids et de la sédentarité était la moins élevée en C.-B. en 2012. Si les taux de prévalence selon l'âge et le sexe de la C.-B. étaient appliqués aux populations des autres provinces, le fardeau économique annuel attribuable à ces facteurs de risque serait réduit de 5,3 milliards, soit $10 \%$ du fardeau économique total de 52,8 milliards de ces facteurs de risque ${ }^{1}$.

Même si la C.-B. possède des taux de prévalence pour ces facteurs de risque moins élevés que toute autre province, il existe d'importants écarts en son sein. La C.-B. est divisée en cinq autorités sanitaires : Fraser Health, Vancouver Coastal Health, Vancouver Island Health, Interior Health et Northern Health. La population des régions sanitaires varie de 0,3 million (Northern Health) à 1,7 million (Fraser Health). Chaque autorité sanitaire est subdivisée en trois ou quatre zones de prestation de services de santé (Health service delivery areas ou HSDA), d'une population variant de 73000 personnes (HSDA de Northeast) à 748000 personnes (HSDA de Fraser South).

Notre étude vise à déterminer la portée d'une éventuelle réduction du fardeau économique en C.-B. si toutes les HSDA de la province atteignaient des taux de prévalence du surplus de poids, de la sédentarité et de l'usage du tabac équivalents à ceux de la HSDA dont les taux sont les plus bas.

\section{Méthodologie}

Des détails de notre modèle de base, ainsi qu'une mise à jour, ont été décrits précédemment $^{1,2,4}$. En bref, nous avons utilisé une approche fondée sur la fraction étiologique du risque pour estimer le fardeau économique associé à ces trois facteurs de risque. Il s'agit de suivre les sept étapes suivantes :

(1) estimer la prévalence des trois facteurs de risque dans les régions géographiques présentant de l'intérêt;

(2) estimer la relation de cause à effet entre le facteur de risque et la comorbidité en se fondant sur le risque relatif;

(3) calculer la fraction étiologique du risque en tenant compte du caractère permanent du surplus de poids (allant de l'absence d'excès de poids au surplus de poids à l'obésité) et de l'usage du tabac (allant de l'absence de tabagisme au tabagisme léger, au tabagisme modéré, au tabagisme lourd);

(4) estimer les coûts directs du traitement des comorbidités associées aux facteurs de risque dans les régions géographiques présentant de l'intérêt;

(5) ajuster les coûts directs associés au chevauchement des facteurs de risque pour une personne donnée;

(6) estimer les coûts indirects;

(7) subdiviser le fardeau économique total pour fournir une estimation du fardeau économique de chaque facteur de risque.

Les taux de prévalence pour l'usage du tabac, le surplus de poids et l'obésité ainsi que la sédentarité ont été tirés de l'Enquête sur la santé dans les collectivités canadiennes (ESCC) de 2011-2012. Les répondants étaient considérés en surpoids si leur indice de masse corporelle (IMC), calculé selon la taille et le poids autodéclarés, se situait entre $25 \mathrm{~kg} / \mathrm{m}^{2}$ et $29,9 \mathrm{~kg} / \mathrm{m}^{2}$ et ils étaient considérés comme obèses si leur IMC était égal ou supérieur à $30 \mathrm{~kg} / \mathrm{m}^{2}$. Pour les jeunes de 12 à 17 ans, le système de classification de Cole pour l'IMC a été utilisé pour déterminer les taux de surplus de poids et d'obésité ${ }^{5}$.

Les fumeurs ont été regroupés comme fumeurs légers (moins de 10 cigarettes par jour), fumeurs modérés (10 à 19 cigarettes par jour) et gros fumeurs (20 cigarettes ou plus par jour) en fonction du nombre moyen de cigarettes fumées par jour selon le fichier de microdonnées à grande diffusion (FMGD) de l'ESCC de 2011-2012 ${ }^{6}$. Tous les fumeurs s'étant définis comme fumeurs occasionnels ont été inclus dans la catégorie des fumeurs légers.
Les taux de sédentarité sont fondés sur les répondants catégorisés comme « pas du tout actifs » dans l'ESCC. Les répondants ont été classés comme étant actifs, modérément actifs ou pas du tout actifs selon un indice d'activité physique quotidienne moyenne pendant les loisirs au cours des trois mois précédents. Pour chaque activité physique déclarée par le répondant, on a calculé une dépense quotidienne moyenne d'énergie en multipliant la fréquence par la durée moyenne de l'activité et par le nombre d'équivalents métaboliques (kilocalories brûlées par kilogramme de poids par heure) de l'activité. L'indice était la somme des dépenses quotidiennes moyennes d'énergie de toutes les activités. Les répondants ont été classés comme étant pas du tout actifs si leur dépense d'énergie pendant les loisirs était inférieure à $1,5 \mathrm{kcal} / \mathrm{kg} /$ jour. Nous avons fait un ajustement à ces données de base de l'ESCC, en estimant les taux de surplus de poids, d'obésité et de sédentarité des enfants âgés de moins de 12 ans en nous fondant sur les taux selon le sexe des 12 à 14 ans de l'ESCC. Nous avons supposé qu'aucun enfant âgé de moins de 12 ans ne fumait.

Les sources et les valeurs pour les risques relatifs associés à l'usage du tabac ${ }^{7}$, au surplus de poids $^{8}$ et à la sédentarité ${ }^{9}$ demeurent les mêmes que pour le modèle précédemment publiée ${ }^{1,2,4}$.

\section{Calcul et ajustement des coûts}

Nous avons estimé le fardeau économique (coûts directs et indirects) associé aux facteurs de risque en C.-B. et dans chaque autorité sanitaire/HSDA de la province à l'aide de la méthode du coût de la maladie fondée sur la prévalence. Les estimations de coûts sont exprimées en dollars canadiens de 2013.

Dans notre modèle, les coûts directs incluent les soins hospitaliers, les services fournis par un médecin ou d'autres professionnels de la santé (à l'exclusion des services dentaires), les médicaments, les coûts liés à la recherche en santé, à la santé publique, à l'administration et aux " autres » dépenses liées à la santé. En C.-B., ces coûts correspondent à 22 milliards du total des 27,1 milliards en dépenses de soins de santé, d'après les données extraites de la Base de 


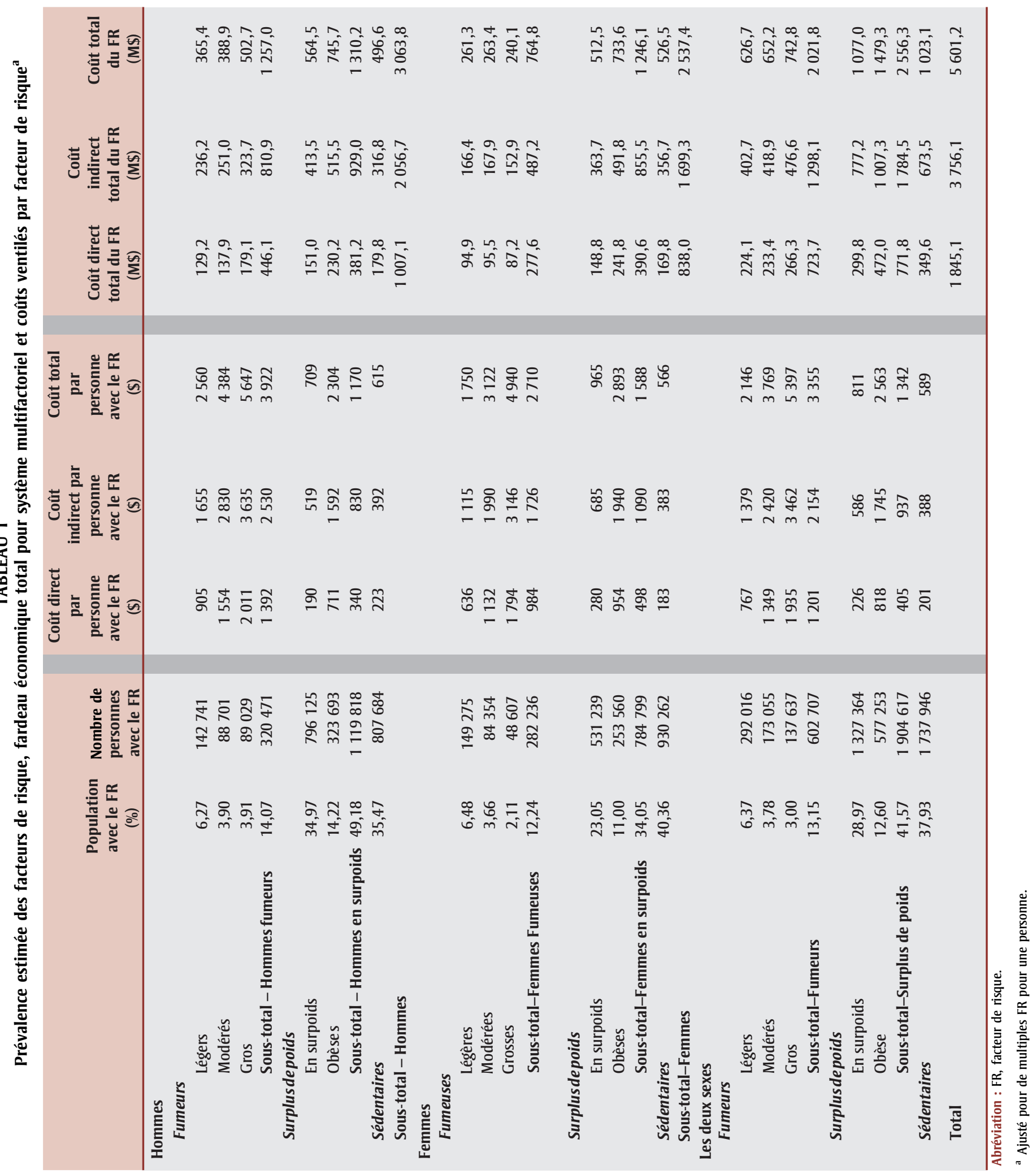


TABLEAU 2

Prévalence estimée des facteurs de risque, fardeau économique total pour système multifactoriel et coûts ventilés par facteur de risque, autorités sanitaires de Colombie-Britannique, 2013, selon le sexe et ajusté pour de multiples facteurs de risque pour une personne

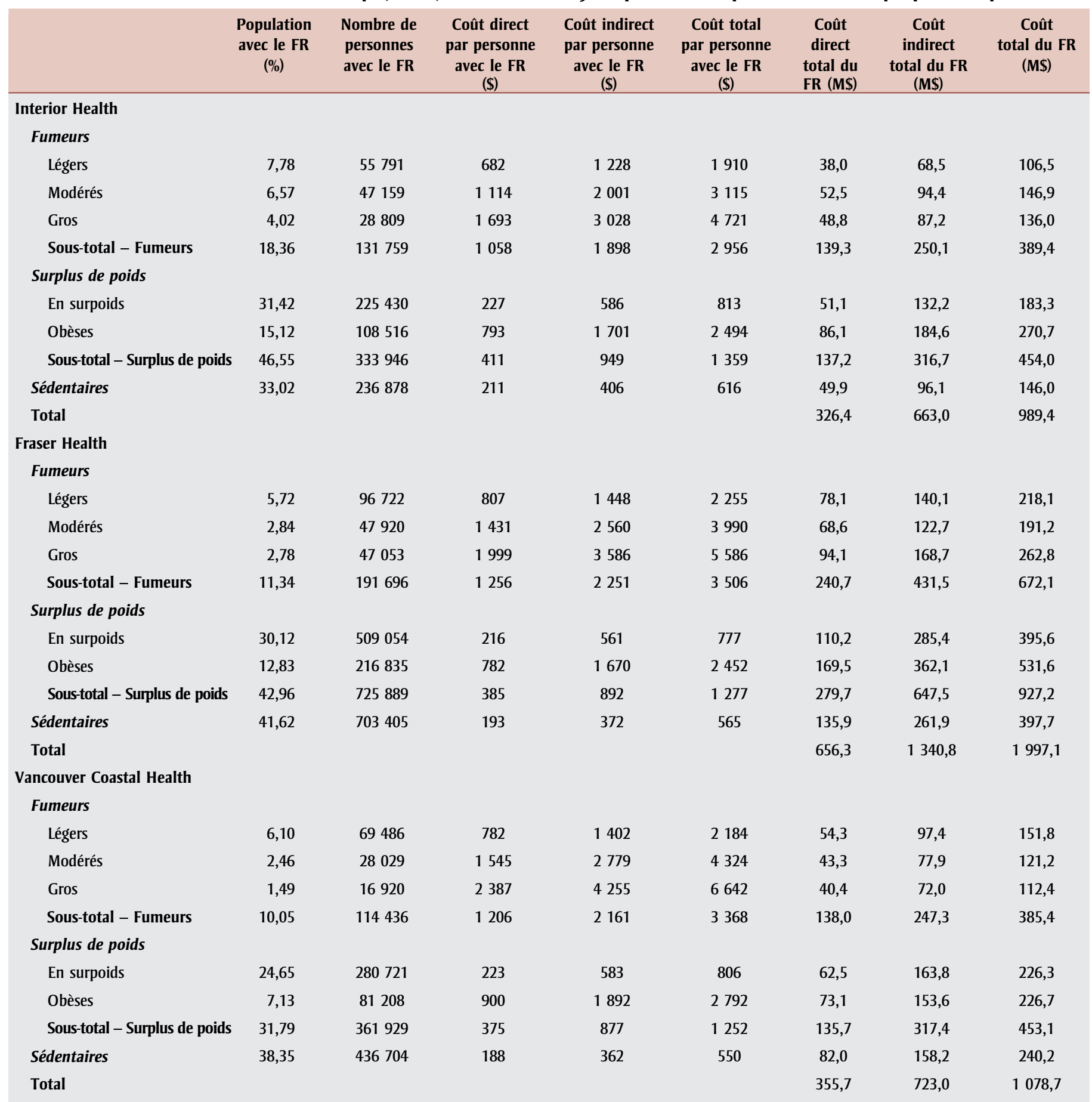

Suite page suivante 
TABLEAU 2 (suite)

Prévalence estimée des facteurs de risque, fardeau économique total pour système multifactoriel et coûts ventilés par facteur de risque, autorités sanitaires de Colombie-Britannique, 2013, selon le sexe et ajusté pour de multiples facteurs de risque pour une personne

\begin{tabular}{|c|c|c|c|c|c|c|c|c|}
\hline & $\begin{array}{c}\text { Population } \\
\text { avec le FR } \\
(\%)\end{array}$ & $\begin{array}{l}\text { Nombre de } \\
\text { personnes } \\
\text { avec le FR }\end{array}$ & $\begin{array}{l}\text { Coût direct } \\
\text { par personne } \\
\text { avec le FR } \\
\text { (\$) }\end{array}$ & $\begin{array}{l}\text { Coût indirect } \\
\text { par personne } \\
\text { avec le FR } \\
\text { (\$) }\end{array}$ & $\begin{array}{l}\text { Coût total } \\
\text { par personne } \\
\text { avec le FR } \\
(\$)\end{array}$ & $\begin{array}{c}\text { Coût } \\
\text { direct } \\
\text { total du } \\
\text { FR (MS) }\end{array}$ & $\begin{array}{c}\text { Coût } \\
\text { indirect } \\
\text { total du FR } \\
\text { (MS) }\end{array}$ & $\begin{array}{l}\text { Coût } \\
\text { total du FR } \\
\text { (MS) }\end{array}$ \\
\hline \multicolumn{9}{|l|}{ Fumeurs } \\
\hline Légers & 6,67 & 50179 & 827 & 1489 & 2316 & 41,5 & 74,7 & 116,2 \\
\hline Modérés & 4,57 & 34377 & 1363 & 2444 & 3807 & 46,9 & 84,0 & 130,9 \\
\hline Sous-total - Fumeurs & 14,59 & 109752 & 1260 & 2258 & 3518 & 138,3 & 247,9 & 386,1 \\
\hline \multicolumn{9}{|l|}{ Surplus de poids } \\
\hline Surpoids & 28,86 & 217084 & 251 & 649 & 901 & 54,5 & 141,0 & 195,5 \\
\hline Obèses & 14,99 & 112728 & 862 & 1849 & 2711 & 97,2 & 208,4 & 305,6 \\
\hline Sous-total - Surplus de poids & 43,85 & 329812 & 460 & 1059 & 1519 & 151,7 & 349,4 & 501,1 \\
\hline \multicolumn{9}{|l|}{ Fumeurs } \\
\hline Légers & 7,10 & 20154 & 749 & 1352 & 2101 & 15,1 & 27,3 & 42,4 \\
\hline Modérés & 5,95 & 16902 & 1172 & 2101 & 3273 & 19,8 & 35,5 & 55,3 \\
\hline Gros & 7,17 & 20357 & 1406 & 2518 & 3924 & 28,6 & 51,3 & 79,9 \\
\hline Sous-total - Fumeurs & 20,23 & 57413 & 1106 & 1986 & 3092 & 63,5 & 114,0 & 177,5 \\
\hline \multicolumn{9}{|l|}{ Surplus de poids } \\
\hline Surpoids & 31,61 & 89713 & 234 & 602 & 836 & 21,0 & 54,0 & 75,0 \\
\hline Obèses & 18,72 & 53135 & 781 & 1683 & 2464 & 41,5 & 89,4 & 130,9 \\
\hline Sous-total - Surplus de poids & 50,33 & 142847 & 437 & 1004 & 1441 & 62,5 & 143,4 & 205,9 \\
\hline
\end{tabular}

Abréviation : FR, facteur de risque.

données sur les dépenses nationales de santé $^{10}$. Les coûts exclus du montant de 27,1 milliards se rapportaient à d'autres établissements ${ }^{*}$ (1,7 milliard), aux services dentaires (2,1 milliards) et aux immobilisations (1,3 milliard).

Les dépenses dans les catégories « autres professionnels de la santé " (soins dentaires, soins de la vue, autres) et « autres dépenses liées à la santé » (recherche et autre) n’ont pas été détaillées pour la C.-B. Nous avons assumé une distribution de ces dépenses équivalente à la distribution au Canada. Pour distribuer ces 27,1 milliards de dollars aux autorités sanitaires et aux HSDA de la C.-B., nous avons d'abord dérivé le volume des cas de soins de courte durée et des jours par autorité sanitaire et HSDA en fonction du lieu de résidence du patient ${ }^{11}$. Ainsi, nous avons attribué les jours passés par un patient dans un hôpital d'une autre région à la région d'où vient le patient. Nous avons ensuite utilisé la distribution des jours-patients en soins de courte durée par autorité sanitaire et HSDA pour distribuer les 8,2 milliards en dépenses pour les hôpitaux en C.-B. Nous avons distribué tous les autres coûts aux autorités sanitaires et aux HSDA en nous fondant sur la proportion des coûts hospitaliers attribués à cette région.
Les soins hospitaliers, les soins fournis par un médecin et les coûts des médicaments selon le sexe ont été attribués à chaque comorbidité d'après les données de 2008 tirées de l'outil en ligne Le fardeau économique de la maladie au Canada (FEMC) ${ }^{12}$. Les comorbidités associées au surplus de poids comprennent certains cancers (cancer de l'œsophage [CIM-10 code C15], cancer colorectal [C18-20], cancer du pancréas [C25], cancer du sein postménopausal [C50], cancer du corps utérin, y compris de l'endomètre [C54-55], des ovaires [C56] et du rein [C64]), le diabète de type 2 (E1114), l'hypertension (I10-15), la cardiopathie ischémique (I20-25), l'embolie pulmonaire

"Il s'agit d'établissements de soins pour bénéficiaires internes souffrant de maladies chroniques ou handicapés qui habitent à l'établissement de façon plus ou moins permanente. 
(I26), la maladie cérébrovasculaire (I60-69), l'asthme (J45), la maladie de la vésicule biliaire (K80-82), l'arthrose (M15-19) et la lombalgie chronique (M45-54). Les comorbidités associées à la sédentarité comprennent le cancer colorectal (C18-20), le cancer du sein (C50), le diabète de type 2 (E11-14), l'hypertension (I10-15), la cardiopathie ischémique (I20-25), la maladie cérébrovasculaire (I60-69) et l'ostéoporose (M80-82). Les comorbidités associées à l'usage du tabac comprennent les cancers des lèvres, de la cavité buccale, du pharynx, du larynx (C00-14, 30-32), de l'œsophage (C15), de l'estomac (C16), colorectal (C18-20), du foie (C22), du pancréas (C25), de la trachée, des bronches, des poumons (C33-34), du rein (C64) et de la vessie (C67) ainsi que la cardiopathie ischémique (I20-25), l'embolie pulmonaire (I26), la thrombo-embolie veineuse (I80-82), la maladie cérébrovasculaire (I60-69), l'anévrisme aortique (I71), la pneumonie (J12-18), la maladie pulmonaire chronique (J40-44), l'ischémie intestinale (K05) et la cirrhose du foie $(\mathrm{K} 70,74)$.

Les données sur les coûts du FEMC n'étaient pas suffisamment détaillées pour certaines de ces comorbidités, dont le diabète de type 2 (E11-14), l'embolie pulmonaire (I26), l'anévrisme aortique (I71), la thrombo-embolie veineuse (I8082), l'ischémie intestinale (K55), la maladie de la vésicule biliaire (K80-82) et la lombalgie chronique (M45-54). Dans chacune de ces situations, nous avons estimé les coûts en nous fondant sur la proportion de journées de soins actifs en milieu hospitalier en 2011-2012 pour la maladie visée par la comorbidité pertinente avec les coûts de 2008 du FEMC. Par exemple, les journées d'hospitalisation pour la lombalgie chronique (M45-54) représentent $21,6 \%$ et $19,6 \%$ (pour les hommes et les femmes, respectivement) de l'ensemble des journées d'hospitalisation pour des maladies du système musculosquelettique et du tissu conjonctif (M00-99) au Canada en 2011-2012. Nous avons donc assumé que $21,6 \%$ et $19,6 \%$ des coûts de 2008 du FEMC attribués à des maladies du système musculosquelettique et du tissu conjonctif (M00-99) pour les soins hospitaliers, les soins fournis par un médecin et les médicaments seraient attribués à la lombalgie chronique (M45-54).
Ces coûts directement liés aux soins selon le sexe par comorbidité ont ensuite été multipliés par les fractions étiologiques du risque calculé selon le facteur, selon le sexe et selon la comorbidité pour calculer les coûts directs des soins attribuables à un facteur de risque donné. Nous avons ajusté ces coûts directs dans un système multifactoriel pour éviter le double comptage (décrit précédemment ${ }^{4}$ ).

\section{Coûts indirects}

Nous avons calculé les coûts indirects (la mortalité prématurée, l'invalidité à court et à long terme) en nous servant de la méthode utilisée dans le FEMC de 1998 (une méthode du capital humain modifiée) ${ }^{13}$. Les coûts indirects attribuables à la mortalité prématurée sont fondés sur la valeur actualisée de la production future perdue, incluant l'évaluation du travail rémunéré et non rémunéré. Les coûts indirects attribuables à l'invalidité à court et à long terme sont également fondés sur la production perdue, en tenant compte de la gravité et de la durée de l'invalidité. L'invalidité à court terme est définie comme une réduction des activités dont la durée prévue est de moins de six mois.

Plus particulièrement, les étapes se rapportant à l'estimation des coûts indirects sont les suivantes :

(1) Les catégories de diagnostics du FEMC de 1998 qui se rapportent aux comorbidités et aux maladies présentant un intérêt ont été déterminées, et les coûts directs et indirects pour ces catégories ont été extraits.

(2) Les coûts extraits ont été utilisés pour établir un ratio entre les coûts directs et indirects pour chacune des catégories de diagnostics, stratifié en fonction de la catégorie précise de coût indirect (p. ex. invalidité à court terme, invalidité à long terme et mortalité prématurée).

(3) Les ratios pertinents (par catégorie de diagnostics et catégorie de coût indirect précis) ont été appliqués aux coûts directs précédemment déterminés attribuables à chaque facteur de risque pour générer les données sur le coût indirect équivalent.
Analyse de la réduction du facteur de risque au niveau des autorités sanitaires et des HSDA

Après avoir calculé le fardeau économique ajusté attribuable aux trois facteurs de risque en C.-B. et dans chaque autorité sanitaire et HSDA, nous avons déterminé quelle région de la province possède la plus faible prévalence globale pour chaque facteur de risque. Les taux de prévalence selon le sexe et l'âge pour chacune des trois régions possédant la plus faible prévalence ont été appliqués à la population de chacune des autres régions. Cela nous a permis de calculer la différence du fardeau économique annuel pour chaque région à l'aide des taux de prévalence réels et des taux de prévalence hypothétiques de ceux des régions de comparaison.

\section{Résultats}

Nous avons estimé le fardeau économique attribuable au surplus de poids, à l'usage du tabac et à la sédentarité en C.-B. en 2013 à 5,6 milliards de dollars, soit un montant de 2,6 milliards de dollars (45,6 \%) attribuable au surplus de poids, 2 milliards de dollars à l'usage du tabac (36,1 \%) et 1 milliard de dollars (18,3\%) à la sédentarité (tableau 1).

Le fardeau économique annuel par personne attribuable au facteur de risque est plus élevé pour tous les fumeurs (3 $355 \$$ ), mais varie de 2146 \$ pour les fumeurs légers à 5397 \$ pour les gros fumeurs. Le fardeau économique annuel par personne en surpoids est de 1342 \$ (811 \$ par personne en surpoids et $2563 \$$ par personne obèse). Même si le fardeau économique annuel par personne en surpoids est beaucoup moins élevé que le fardeau par fumeur, la prévalence élevée du surplus de poids $(41,6 \%)$ comparativement à la prévalence de l'usage du tabac (13,2\%) en C.-B. signifie que le fardeau économique annuel total attribuable au surpoids dépasse maintenant celui attribuable à l'usage du tabac de $26 \%$.

À l'échelle des autorités sanitaires, la prévalence de l'usage du tabac était plus élevée que la moyenne provinciale dans Interior Health, Northern Health et 
Vancouver Island Health, et la prévalence du surplus de poids était plus élevée que la moyenne provinciale dans toutes les autorités sanitaires à l'exception de Vancouver Coastal Health. Inversement, la prévalence de la sédentarité était plus faible que la moyenne provinciale dans Interior Health et dans Vancouver Island Health. La prévalence des trois facteurs de risque était supérieure à la moyenne provinciale dans Northern Health (tableau 2). Le fardeau économique total attribuable à ces trois facteurs de risque dans les autorités sanitaires variait de 453 millions de dollars dans Northern Health à 1997,1 millions de dollars dans Fraser Health.

La prévalence du surplus de poids varie selon la HSDA, allant de 29,5\% dans la HSDA de Vancouver à 56,7 \% dans la HSDA Northwest (moyenne en C.-B. de $41,6 \%$, voir la figure 1 ). La prévalence de la sédentarité varie de $27,1 \%$ dans la HSDA de Kootenay Boundary à 43,8 \% dans la HSDA de Fraser North (moyenne en C.-B. de 37,9 \%, voir la figure 2). La prévalence de l'usage du tabac varie de 8,8 \% dans la HSDA de Richmond à 21,3\% dans la HSDA Northwest (moyenne en C.-B. de $13,2 \%$, voir la figure 3 ).

Les taux de prévalence variables des trois facteurs de risque dans chacune des HSDA se traduisent par un fardeau économique par personne variable dans chacune des régions (voir la figure 4). La HSDA de Richmond possède le fardeau économique par personne le moins élevé à 738 \$, tandis que la HSDA Northwest a le plus élevé à 1766 \$, plus du double de celui de la HSDA de Richmond. La moyenne provinciale du fardeau économique par personne s'élève à 1222 \$.

L'application des taux de prévalence les plus bas selon le sexe et l'âge pour le surplus de poids de la HSDA de Vancouver, pour l'usage du tabac de la HSDA de Richmond et pour la sédentarité de la HSDA de Kootenay Boundary à la population des autres HSDA de la province réduirait le fardeau économique annuel par personne d'un montant entre 60 \$ (pour la HSDA de Richmond) et 651 \$ (pour la HSDA Northwest) (voir la figure 5).

La réduction annuelle totale du fardeau économique varierait entre 12,1 millions de dollars pour la HSDA de Richmond et 200,1 millions de dollars pour la HSDA Fraser South (voir la figure 6). Si toutes les HSDA devaient atteindre les meilleurs taux de prévalence pour les trois facteurs de risque, un fardeau économique de 1362,2 millions de dollars pourrait être évité annuellement, soit 449,8 millions de dollars en coûts directs et 912,4 millions de dollars en coûts indirects.

\section{Analyse}

Nous avons estimé le fardeau économique annuel attribuable au surplus de poids, à l'usage du tabac et à la sédentarité en C.-B. à 5,6 milliards de dollars en 2013, avec une proportion plus élevée de ce total attribuable au surplus de poids (2,6 milliards de dollars) qu'à l'usage du tabac (2 milliards de dollars). Même si la C.-B. a des taux de prévalence des facteurs de risque plus faibles que n'importe quelle autre province canadienne ${ }^{1}$, ces taux varient beaucoup au sein de la province. Si chaque HSDA de la province atteignait les meilleurs taux de prévalence pour les trois facteurs de risque, un fardeau économique de 1,36 milliard de dollars pourrait être évité annuellement. Cela donne à penser qu'une réduction de $24 \%$ du fardeau économique attribuable au surplus de poids, à l'usage du tabac et à la sédentarité en C.-B. est possible si toutes les régions atteignaient les taux pour ces facteurs de risque qui sont les meilleurs de la province. Cependant, il est important de noter qu'une réduction du fardeau économique n'équivaut pas à des économies de coûts. Même pour les coûts directs, la majorité des ressources dégagées au fil du temps seront vraisemblablement réaffectées (intentionnellement ou non) ailleurs dans le domaine des soins de santé.

Une analyse semblable utilisant les taux de prévalence selon l'âge et le sexe en C.-B. appliqués aux populations vivant dans les autres provinces canadiennes a révélé que le fardeau économique annuel au Canada attribuable à ces trois facteurs de risque serait réduit de 5,3 milliards de dollars, soit $10 \%$ du fardeau économique total de 52,8 milliards de dollars des facteurs de risque $^{1}$. La variation au sein des provinces de la prévalence des facteurs de risque semble donc beaucoup plus grande que la variation entre les provinces.

\section{FIGURE 1 \\ Prévalence du surplus de poids en Colombie-Britannique, par catégorie de surplus de poids et HSDA, 2011-2012}

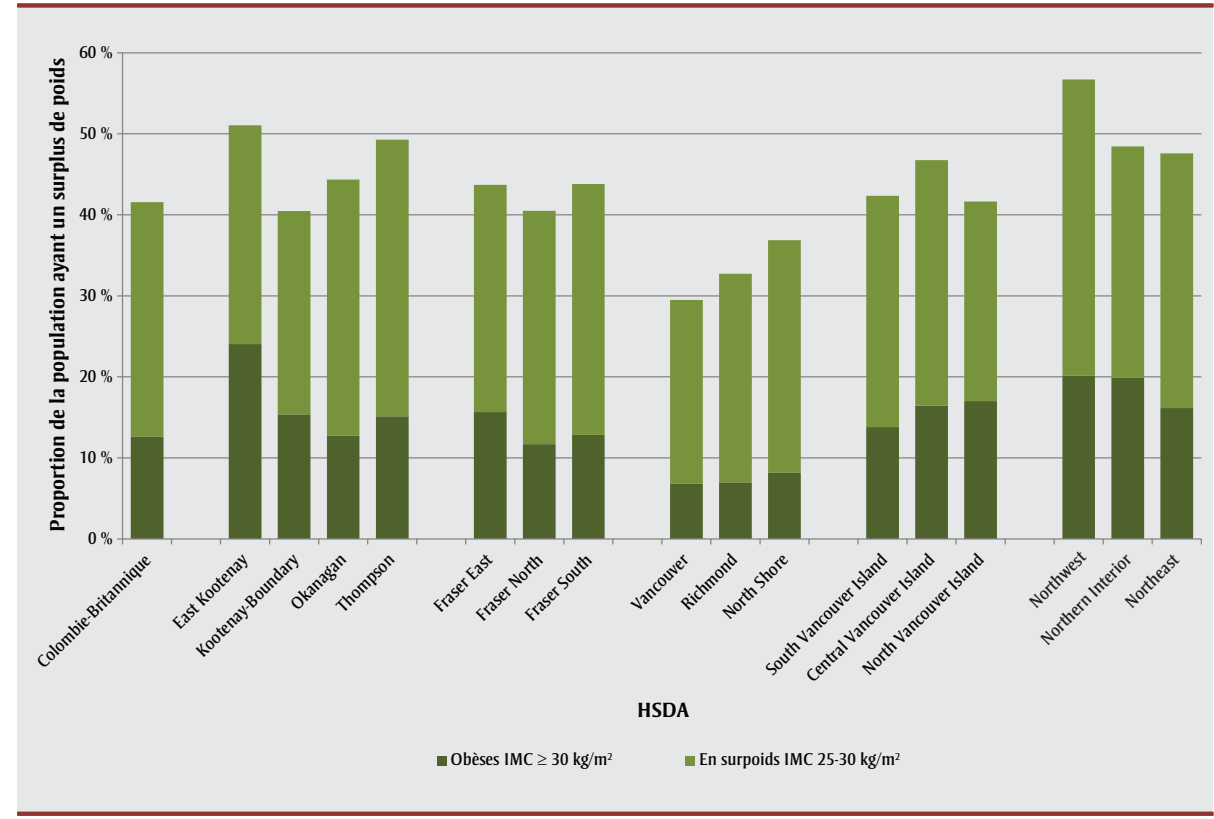

Abréviations : HSDA, health service delivery area (zone de prestation de services de santé); IMC, indice de masse corporelle. 
FIGURE 2

Prévalence de la sédentarité en Colombie-Britannique, par HSDA, 2011-2012

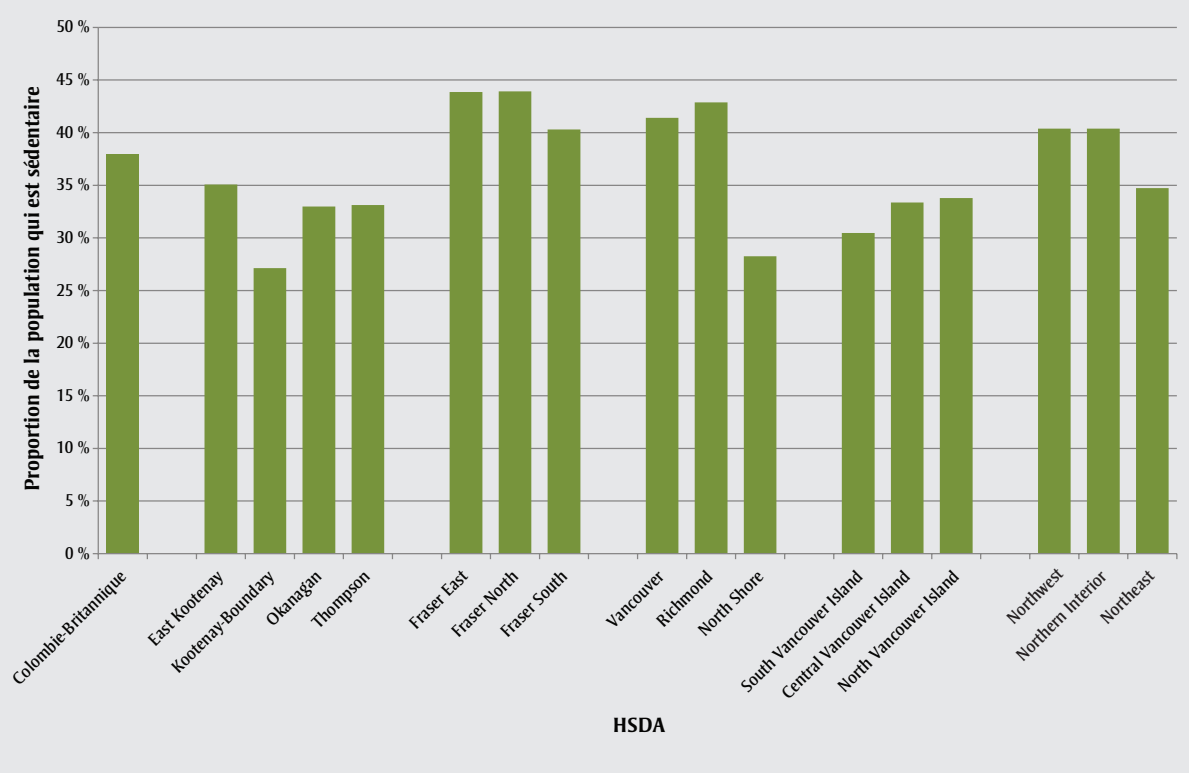

Abréviation : HSDA, health service delivery area (zone de prestation de services de santé).

Cette étude a permis de cerner d'importantes disparités dans la prévalence de chacun des facteurs de risque entre les régions sanitaires, qui se sont reflétées dans le fardeau économique attribuable de cha-

FIGURE 3

Prévalence de l'usage du tabac en Colombie-Britannique, par intensité du tabagisme et HSDA, 2011-2012

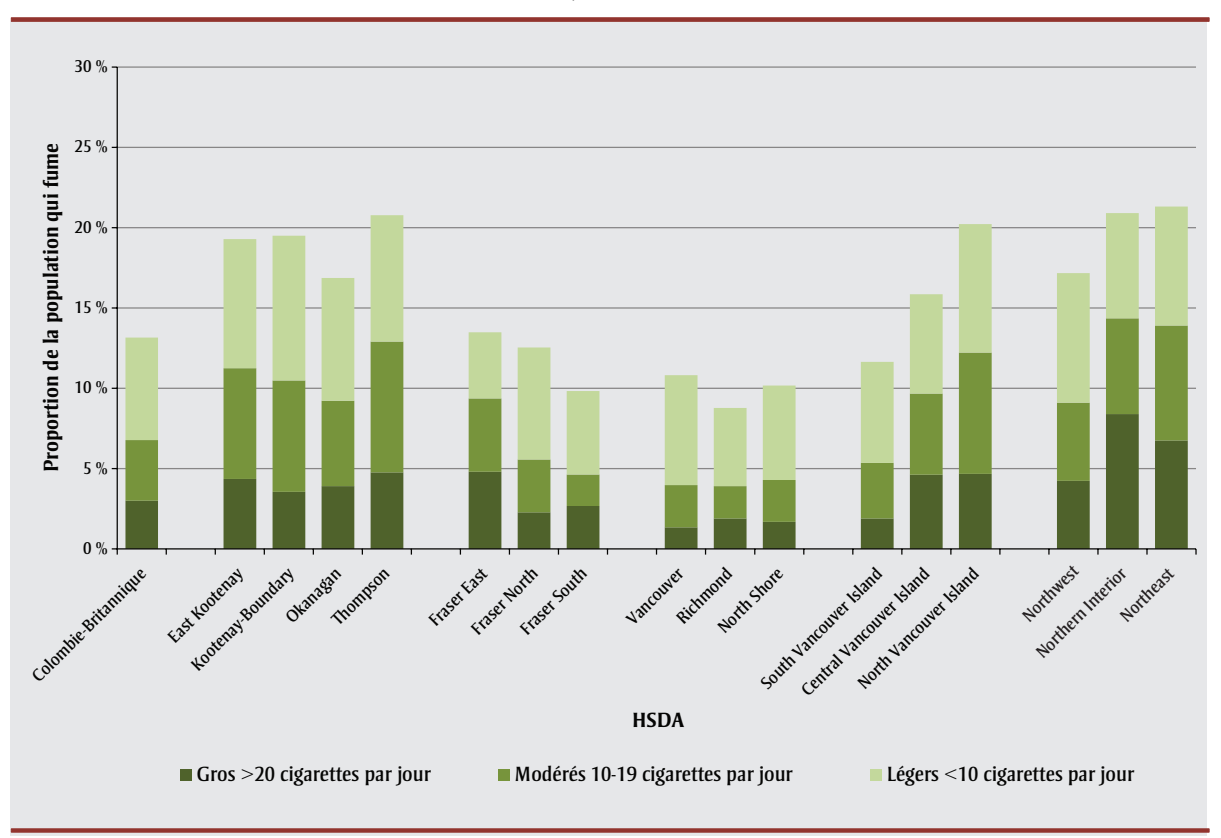

Abréviation : HSDA, health service delivery area (zone de prestation de services de santé). étaient généralement beaucoup plus élevés dans les régions ayant une densité de population plus élevée (en particulier dans les HSDA de Fraser North, de Fraser East, de Vancouver et de Richmond) par rapport aux populations des zones plus rurales. Inversement, les taux de tabagisme étaient beaucoup moins élevés dans les régions urbaines par rapport aux régions rurales. Les taux des facteurs de risque étaient presque toujours supérieurs à la moyenne provinciale dans le nord de la province (HSDA de Northwest, de Northern Interior et de Northeast).

Certains allèguent que l'épidémie d'obésité constitue le « nouveau tabac », en se fondant sur l'augmentation rapide de sa prévalence dans le monde et sur l'ensemble de ses conséquences sur la santé. Les taux de tabagisme ont beaucoup diminué au cours des dernières décennies, et ces progrès devraient nous encourager à espérer que de telles réussites sont aussi possibles pour les autres facteurs de risque modifiables ${ }^{14,15}$. Nous avons appris de notre expérience avec l'usage du tabac qu'une approche exhaustive sur plusieurs fronts est nécessaire pour parvenir à des réductions importantes ${ }^{16}$. La réduction des taux de tabagisme n'est pas uniquement attribuable à une ou deux interventions : il s'agit plutôt de la combinaison d'interventions économiques et politiques, d'interventions communautaires et d'interventions cliniques qui ont agi en synergie afin d'abaisser les taux de tabagisme à leur niveau actuel. Nous avons également appris que pour observer une réduction considérable de la prévalence des facteurs de risque, une approche à long terme est nécessaire. Le problème du tabagisme n'a pas été résolu par une solution rapide, et il semble improbable que d'autres facteurs de risque modifiables le soient. Il faut plutôt favoriser les interventions adoptant des approches multigénérationnelles dont l'envergure dépasse le cycle politique immédiat.

Pour cette étude, nous avons mis l'accent sur les coûts associés aux facteurs de risque de niveau individuel, mais nous reconnaissons également que le surplus de poids, la sédentarité et l'usage du tabac sont fortement influencés par un éventail de facteurs sociaux, environnementaux et économiques. Ces déterminants expliquent probablement une partie des variations géographiques que 
FIGURE 4

Fardeau économique par habitant en Colombie-Britannique : tabagisme, surplus de poids et sédentarité, par HSDA, 2013

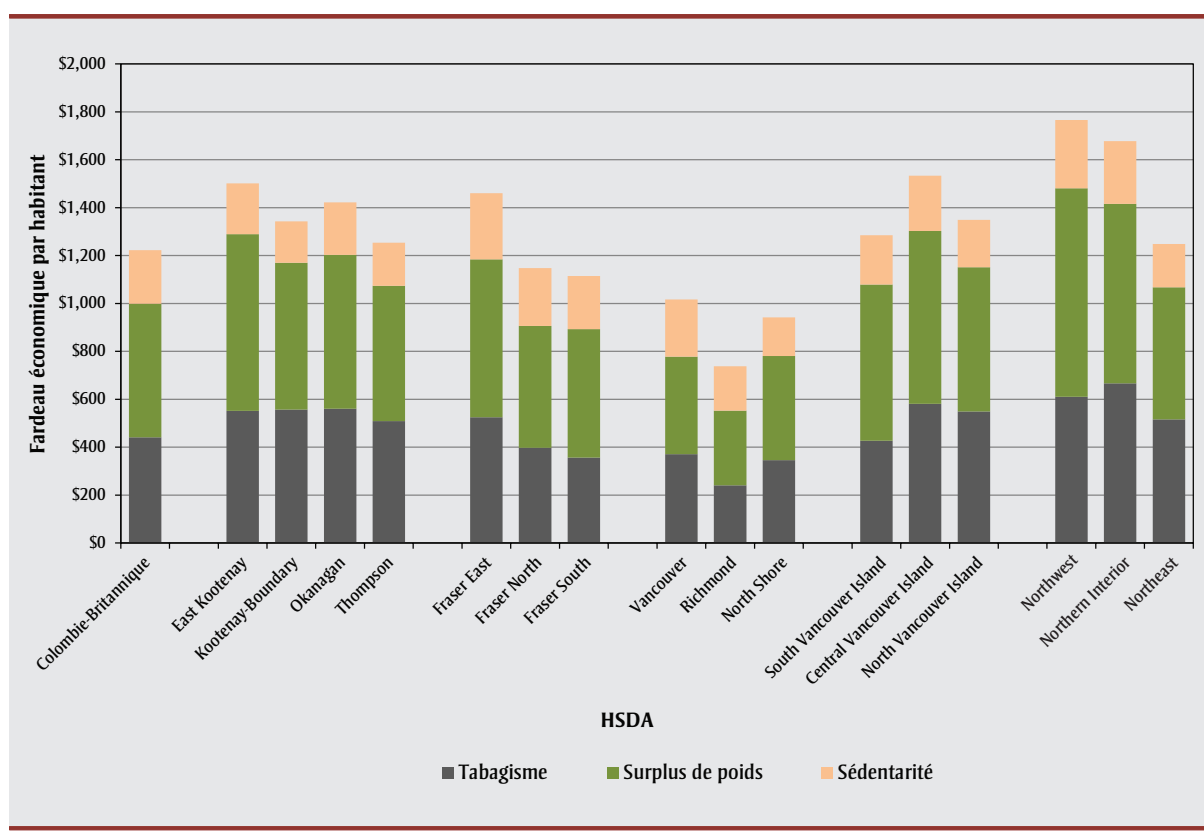

Abréviation : HSDA, health service delivery area (zone de prestation de services de santé).

FIGURE 5

Changements dans le fardeau économique annuel par habitant en Colombie-Britannique, fondés sur les meilleurs taux des facteurs de risque, par HSDA et coûts directs/indirects (en M\$), 2013

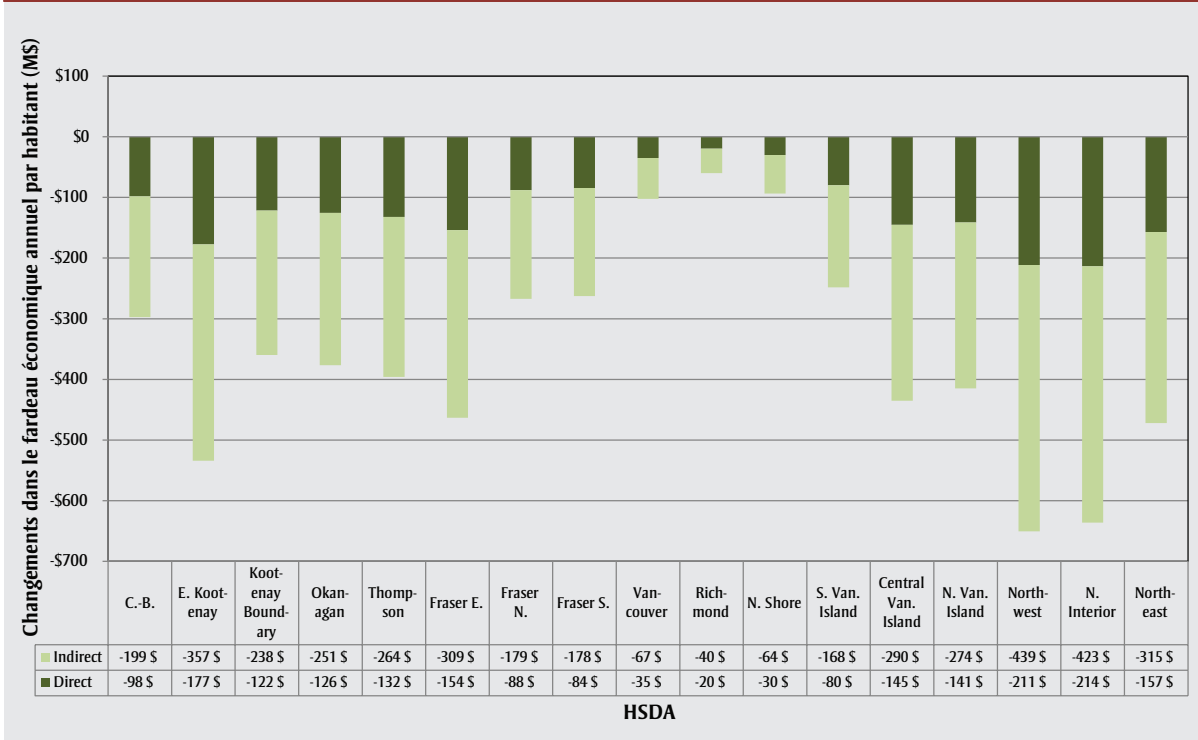

Abréviations : C.-B., Colombie-Britannique; HSDA, health service delivery area (zone de prestation de services de santé).

Remarque : L'analyse de la sensibilité suggère que ces coûts peuvent varier de $+/-17 \%$. nous avons observées, et ces facteurs sousjacents devraient également être pris en compte dans un effort visant à promouvoir l'équité en matière de santé. Tous les citoyens n'ont pas une chance égale de faire des choix sains, et les interventions au niveau de la population devraient tenir compte des facteurs de risque des maladies chroniques tout en reconnaissant les déterminants sociaux de la santé.

L'inclusion des coûts indirects dans n'importe quelle analyse économique est controversée étant donné qu'il existe une grande diversité d'approches, lesquelles produisent des résultats très différents ${ }^{17-20}$. En 1998, le FEMC a utilisé une méthode du capital humain modifiée ${ }^{\dagger}$, passant à la méthode des coûts de friction ${ }^{\dagger}$ en 2008. Les coûts indirects qui en découlent varient sensiblement (voir le tableau 3).

Si la méthode des coûts de friction était appliquée au modèle actuel, le fardeau économique indirect attribuable aux trois facteurs de risque en C.-B. diminuerait, passant de 3756 millions de dollars à 238 millions de dollars. La méthode des coûts de friction met l'accent sur la production perdue [traduction] « du point de vue des entreprises, des consommateurs et de la société, sans tenir compte de la perte de revenu potentielle sur une base individuelle ${ }^{2,}$ p.452, sans non plus accorder de valeur au temps potentiel perdu en raison de la morbidité ou de la mortalité. Donc, même si le tabagisme réduit en moyenne l'espérance de vie d'une personne de 11 à 12 ans $^{22}$, la méthode des coûts de friction n'accorde une valeur qu'à la période de temps nécessaire pour remplacer cette personne sur le marché du travail. Le fait d'accorder une valeur économique au temps perdu en raison de l'invalidité et de la mortalité précoce (comme dans la méthode du capital humain modifiée) nous permet de comparer l'incidence plus générale des facteurs de risque sur la société dans son ensemble, plutôt qu'en mettant l'accent sur les pertes de production.

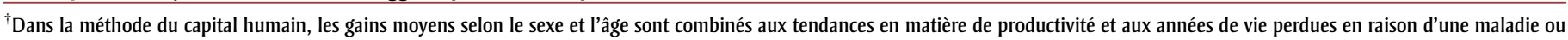

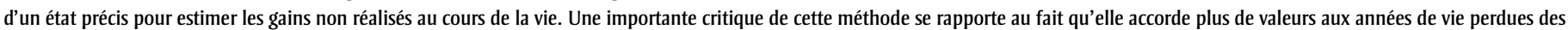

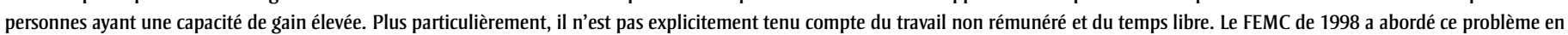
accordant une valeur explicite au temps non productif.

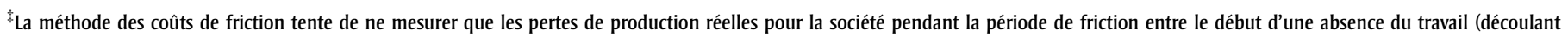
d'une absence à court terme ou à long terme, de l'invalidité et de la mortalité) et lorsque les niveaux de productivité originaux sont rétablis. 
FIGURE 6

Changements dans le fardeau économique annuel par habitant en Colombie-Britannique, fondés sur les meilleurs taux des facteurs de risque, par HSDA et coûts directs/indirects (en M\$), 2013

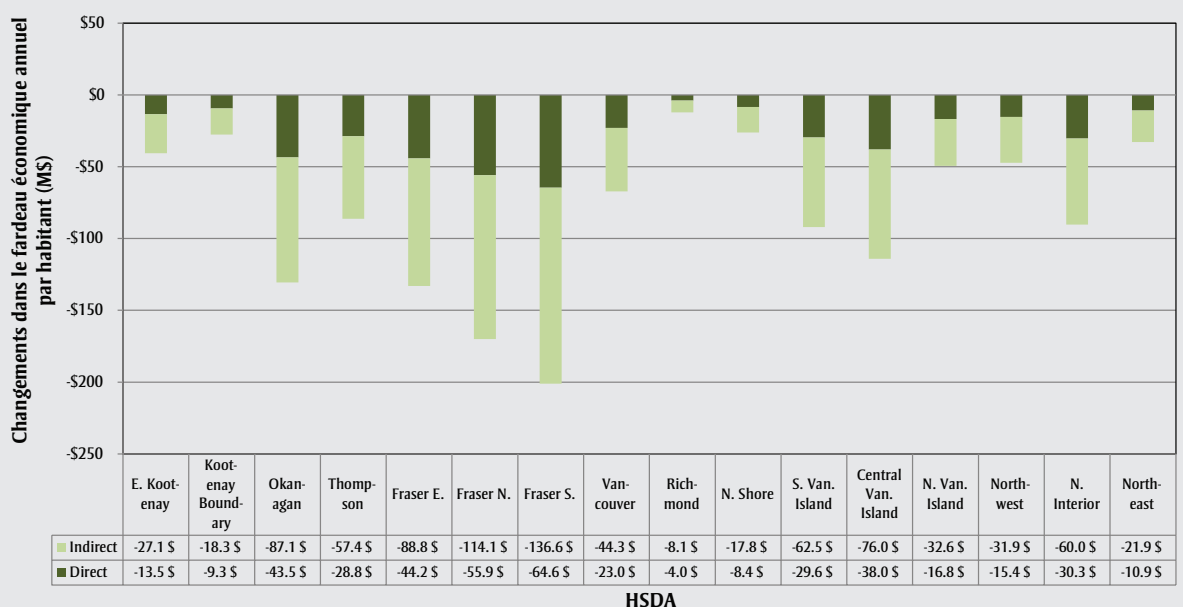

Abréviation : HSDA, health service delivery area (zone de prestation de services de santé). Remarque : L'analyse de la sensibilité suggère que ces coûts peuvent varier de $+/-17 \%$.

\section{Points forts et limites}

Malgré tous les efforts en vue d'optimiser l'exactitude de l'analyse, certaines limites demeurent. La plupart des études, y compris la présente, catégorisent les personnes ayant un IMC entre $25 \mathrm{~kg} / \mathrm{m}^{2}$ et $29,9 \mathrm{~kg} / \mathrm{m}^{2}$ comme étant en surpoids. Cette plage, particulièrement la partie inférieure, a cependant évolué au fil du temps ${ }^{16}$. Les recherches récentes ont suggéré qu'une limite inférieure plus appropriée en ce qui concerne les effets négatifs sur la santé pourrait être fixée à $27 \mathrm{~kg} / \mathrm{m}^{2} 23$, ou même sous la barre des $25 \mathrm{~kg} / \mathrm{m}^{2}$ pour certaines personnes, en particulier celles d'origine asiatique $^{24}$. Ces considérations sont pertinentes pour l'étude actuelle puisqu'une proportion élevée de gens en C.-B. se définissent comme membres d'une minorité ethnique visible $(24,8 \%)^{25}$, certaines régions ayant une proportion beaucoup plus élevée que d'autres. Par exemple, dans la HSDA de Richmond, $44 \%$ des gens se sont déclarés d'origine chinoise, $8 \%$ d'Asie du Sud et 5,5\% des Philippines. L'utilisation d'une limite de l'IMC de $25 \mathrm{~kg} / \mathrm{m}^{2}$ pour cette population est susceptible d'entraîner une sous-estimation du fardeau économique attribuable au surplus de poids.

La méthode consistant à passer des coûts directs aux coûts indirects dépend de l'hypothèse selon laquelle les ratios de coûts n'ont pas changé au fil du temps. De plus, la source pour les risques relatifs associés au tabagisme ${ }^{7}$ et à la sédentaritée ${ }^{9}$ 'ajuste aux facteurs aggravants connus engendrant des risques relatifs propres à une maladie. Les méta-analyses pour les risques relatifs associés au surpoids et à l'obésité n'incluent cependant pas la sédentarité en tant que facteur de risque potentiellement aggravant ${ }^{8}$, ce qui peut mener à une surestimation du fardeau économique attribuable au surplus de poids. D'un autre côté, les risques relatifs calculés dans cette méta-analyse sont fondés sur une combinaison d'études incluant des mesures axées sur l'autodéclaration et des mesures objectives de l'IMC, tandis que notre modèle utilise la prévalence du surplus de poids fondée sur la taille et le poids autodéclarés, ce qui pourrait mener à une sous-estimation du fardeau économique attribuable au surplus de poids. Des analyses de sensibilité menées précédemment suggèrent également que le véritable fardeau économique pourrait varier de plus ou moins $17 \%$ par rapport à notre meilleure estimation ${ }^{2}$. Enfin, l'affectation des coûts liés aux soins non hospitaliers aux autorités sanitaires et aux HSDA en proportion de l'affectation des coûts liés aux soins hospitaliers pourrait entraîner une surestimation ou une sous-estimation de ces coûts dans certaines régions de la province.

\section{Conclusion}

Nos résultats suggèrent que le fardeau économique du surplus de poids, de la sédentarité et de l'usage du tabac est important et varie considérablement parmi les régions sanitaires de la C.-B.

TABLEAU 3

Fardeau économique de la maladie au Canada par catégorie de diagnostic, coûts indirects en tant que pourcentage des coûts directs

\begin{tabular}{|c|c|c|c|c|c|c|}
\hline \multirow[t]{2}{*}{ Catégorie de diagnostic } & \multicolumn{3}{|c|}{ FEMC 1998 (capital humain) } & \multicolumn{3}{|c|}{ FEMC 2008 (friction) } \\
\hline & Mortalité (\%) & Morbidité (\%) & Total (\%) & Mortalité (\%) & Morbidité (\%) & Total $(\%)$ \\
\hline Tumeurs malignes et autres néoplasmes & 431 & 46 & 478 & 3,5 & 8,8 & 12,3 \\
\hline Maladies cardiovasculaires & 121 & 50 & 171 & 0,8 & 2,3 & 3,1 \\
\hline Maladies respiratoires / Infections & 48 & 99 & 146 & 0,3 & 46,8 & 47,1 \\
\hline
\end{tabular}

Abréviation : FEMC, Fardeau économique de la maladie au Canada. 
Cependant, en réduisant la prévalence de chacun des trois facteurs de risque à l'échelle de l'ensemble de la province à celle de la région possédant la prévalence la plus faible, les coûts directs et indirects associés pourraient être réduits d'environ un quart. Sachant cela, la priorisation des initiatives de prévention devrait être au premier plan des changements apportés au niveau du système et des communautés. La preuve économique que nous présentons suggère également que diverses régions de la C.-B. exigent une attention particulière. Plus particulièrement, les variations spatiales entre les autorités sanitaires et les HSDA pourraient servir de ligne directrice pour déterminer là où les efforts de prévention ciblant une région en particulier sont les plus utiles. Différents facteurs sociaux, environnementaux et économiques expliquent probablement une partie de ces variations spatiales, et ces facteurs sousjacents devraient être pris en considération lors de l'élaboration de programmes de prévention visant à promouvoir l'équité en matière de santé.

\section{Remerciements}

Cette étude a été menée dans le cadre d'un plan de travail conjoint des programmes de surveillance de la santé de la population entre le ministère de la Santé et la Provincial Health Services Authority (PHSA). Les auteurs remercient Lydia Drasic (directrice exécutive, BCCDC Operations and Chronic Disease Prevention, PHSA) et Silas Brownsey (directeur exécutif, Population and Public Health, BC Ministry of Health) d'avoir parrainé ce projet d'étude.

\section{Références}

1. Krueger H, Krueger J, Koot J. Variation across Canada in the economic burden attributable to excess weight, tobacco smoking and physical inactivity. Can J Public Health. 2015;106(4):e171-177. DOI: 10.17269/cjph. 106.4994 .

2. Krueger H, Turner D, Krueger J, Ready AE. The economic benefits of risk factor reduction in Canada: tobacco smoking, excess weight and physical inactivity. Can J Public Health. 2014;105(1):e69-78.
3. Statistique Canada. CANSIM [Internet]. Ottawa (Ont.) : Statistique Canada; [consultation le 29 sept. 2014]. Consultable en ligne à la page: http://www5.statcan.gc.ca/ cansim/

4. Krueger H, Williams D, Ready AE, Trenaman L, Turner D. Meilleure estimation du fardeau que représentent les facteurs de risque de maladie chronique pour la santé et l'économie au Manitoba. Maladies chroniques et blessures au Canada, 2013;33(4):236-246.

5. Cole TJ, Bellizzi MC, Flegal KM, Dietz WH. Establishing a standard definition for child overweight and obesity worldwide: international survey. BMJ. 2000;320(7244):1240-1243.

6. Statistique Canada. Fichier de microdonnées à grande diffusion de l'Enquête sur la santé dans les collectivités canadiennes, 2011-2012 [Internet]. Ottawa (Ont.) : Statistique Canada; [modifié le 13 nov. 2013]. [Statistique Canada, $\mathrm{n}^{\mathrm{o}}$ de Catalogue : 82M0013X2013001]. Consultable en ligne à la page: http://www5. statcan.gc.ca/olc-cel/olc.action?ObjId $=82 \mathrm{M}$ 0013 X2013001\&ObjType $=46 \&$ lang $=$ fr\& limit $=0$

7. Pirie K, Peto R, Reeves GK, Green J, Beral V. The $21^{\text {st }}$ century hazards of smoking and benefits of stopping: a prospective study of one million women in the UK. Lancet. 2013. 381(9861)133-141. DOI: 10.1016/S01406736 (12)61720-6.

8. Guh DP, Zhang W, Bansback N, Amarsi Z, Birmingham CL, Anis AH. The incidence of co-morbidities related to obesity and overweight: a systematic review and meta-analysis. BMC Public Health. 2009;9:88. DOI: 10.1186/ 1471-2458-9-88.

9. Katzmarzyk PT, Janssen I. The economic costs associated with physical inactivity and obesity in Canada: an update. Can J Appl Physiol. 2004;29(1):90-115.

10. Kuchciak C, Fortin G, Ariste R. et collab. Groupe d'experts des dépenses nationales de santé. Tendances des dépenses nationales de santé, de 1975 à 2013. Ottawa (Ont.) : Institut canadien d'information sur la santé; 2013.

11. Ministry of Health. HealthIdeas [Internet]. Victoria (BC) : Ministry of Health; [consulté le $1^{\text {er }}$ juillet 2014]; Consultable en ligne à la page: http://public.healthideas.gov.bc. ca/portal/page/portal/HealthIdeas
12. Population Health Economics, Agence de la santé publique du Canada. Le fardeau économique de la maladie au Canada, 20052008 : Protéger les Canadiens des maladies [Internet]. Ottawa (Ont.) : Agence de la santé publique du Canada; [consulté le $1^{\mathrm{er}}$ nov. 2014]. Consultable en ligne à la page: http://ebic-femc.phac-aspc.gc.ca/index.php? lang $=$ fra

13. Division de la recherche sur les politiques, Santé Canada. Le fardeau économique de la maladie au Canada, 1998. Ottawa (Ont.) : Santé Canada; 2002.

14. McLean D, Williams D, Lamont S, Krueger K. Community-based prevention: reducing the risk of cancer \& chronic disease. Toronto (Ont.) : University of Toronto Press; 2013.

15. Record NB, Onion DK, Prior RE et collab. Community-wide cardiovascular disease prevention programs and health outcomes in a rural county, 1970-2010. JAMA. 2015;313(2): 147-55. DOI: 10.1001/jama.2014.16969.

16. Krueger H, Williams D, Kaminsky B, McLean D. The health impact of smoking and obesity and what to do about it Toronto (Ont.) : University of Toronto Press; 2007.

17. Goeree R, O’Brien BJ, Blackhouse F, Agro K, Goering P. The valuation of productivity costs due to premature mortality: a comparison of the human-capital and frictioncost methods for schizophrenia. Can J Psychiatry. 1999;44:455-463.

18. Hutubessy RC, van Tulder MW, Vondeling $\mathrm{H}$, Bouter LM. Indirect costs of back pain in the Netherlands: a comparison of the human capital method with the friction cost method. Pain. 1999;80(1):201-217.

19. Lofland JG, Locklear JC, Frick KD. Different approaches to valuing the lost productivity of patients with migraine. Pharmacoeconomics. 2001;19(9):917-925.

20. Yabroff KR, Bradley CJ, Mariotto AB, Brown ML, Feuer EJ. Estimates and projections of value of life lost from cancer deaths in the United States. J Natl Cancer Inst. 2008; 100(24):1755-1762.

21. Tranmer JE, Guerriere DN, Ungar WJ, Coyte PC. Valuing patient and caregiver time: a review of the literature. Pharmacoeconomics. 2005;23(5):449-459. 
22. Jha P, Ramasundarahettige $\mathrm{C}$, Landsman $\mathrm{V}$ et collab. $21^{\text {st }}$-century hazards of smoking and benefits of cessation in the United States. N Engl J Med. 2013;368(4):341-50. DOI: 10.1056/NEJMsa1211128.

23. Hu FB, Willet WC, Li T, Stampfer MJ, Colditz GA, Manson JE. Adiposity as compared with physical activity in predicting mortality among women. N Engl J Med. 2004;351(26):2694-703.

24. WHO Expert Consultation. Appropriate body-mass index for Asian populations and its implications for policy and intervention strategies. Lancet. 2004;363(9403):157-163.

25. BC Stats socio-economic profiles [Internet]. Victoria (BC): BC Stats [consulté le $1^{\text {er }}$ novembre 2014]. Consultable en ligne à la page. http://www.bcstats.gov.bc.ca/Statistics BySubject/SocialStatistics/SocioEconomic ProfilesIndices/Profiles.aspx 